\title{
Design of Intelligent Monitoring System of Chicken House Environment Based on Single-chip Microcomputer
}

\author{
Qing Du ${ }^{1, a}, Y_{a n h u a ~ M i a o^{1, b}}$ and Yunhui Zhang ${ }^{1, c}$ \\ ${ }^{1}$ Institute of Information and Automation, Tianjin Tianshi College, Tianjin, China \\ aduqing_1982@126.com, bff-peacock@163.com, 'c602272248@qq.com
}

Key words: Environmental monitoring; Chicken house; Single-chip microcomputer

\begin{abstract}
In view of the problem that some chicken farms are susceptible to various bacteria and viruses due to poor breeding environment, this paper designs a chicken house environmental intelligent monitoring system based on single-chip microcomputer application to improve the chicken house environment. The system adopts STC89C52 single-chip microcomputer as the main control chip. The sensor collects information on the light intensity, temperature and humidity, and carbon dioxide concentration, and controls the exhaust fan and the illumination lamp, and the environmental parameters can be displayed on the display in real time.
\end{abstract}

\section{Introduction}

With the continuous development of the society, the current aquaculture industry has also developed rapidly, and many large-scale breeding enterprises and a large number of rural farmers have emerged. In many places, a large-scale farming and one-stop industrial chain has been formed. How to improve the efficiency of enterprises, from the aquaculture industry to obtain profits, is a very concerned issue for each enterprise. In addition to good variety and reasonable nutrition, the environment in which animals grow is also very important. It is very necessary to ensure the environment for the growth and development of the adapted animals in the enclosure and improve the economic benefits through a set of monitoring programs of temperature, humidity and harmful gases.

This paper mainly studies the environment monitoring system of chicken house based on single-chip microcomputer, realizes the monitoring of temperature, humidity, illumination and air circulation. The monitored data is fed back to the monitoring personnel via the LCD. The monitoring system mainly consists of a single-chip control module, an A/D conversion module, a sensor module, an LCD display module, a buzzer alarm module, an execution module, and a power module. The single-chip microcomputer processes the signal collected by the sensor through $\mathrm{A} / \mathrm{D}$ conversion and displays it on the LCD. When the concentration of carbon dioxide exceeds the limit value, the buzzer will alarm and turn on the fan. When the illumination value is lower than the set value, the light will be turned on to ensure the ecological environment in the poultry house is good.

\section{System structure}

The system uses single-chip microcomputer as the main control module of the whole system. The sensor collects the information and LCD displays the data. The system block diagram is shown in Fig. 1. In this design, the carbon dioxide sensor module is used to complete the collection of $\mathrm{CO}_{2}$ concentration, and the photosensitive module completes the collection of the light intensity. The temperature and humidity sensor module uses the DHT11 sensor to collect temperature and humidity information, and then visualizes the data through the LCD1602 liquid crystal display. The alarm module uses buzzer to alarm, and the executive module uses 5V DC fan and LED to control carbon dioxide concentration and light supply. Matrix keyboard can be used to change the limit values of various environmental factors.

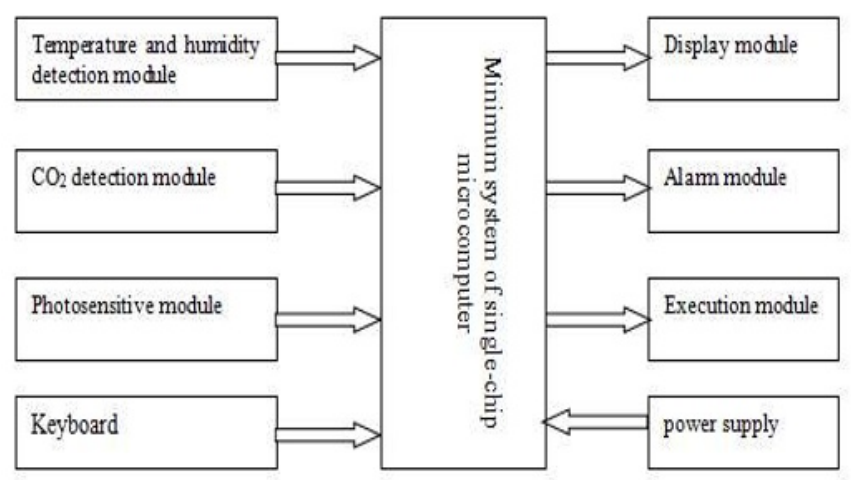

Figure 1. System block diagram 


\section{System hardware design}

\subsection{Minimum system of single-chip microcomputer}

The minimum system of the single-chip microcomputer mainly includes the reset circuit and the crystal oscillator circuit. The schematic diagram of the minimum system hardware composition of the single-chip microcomputer is shown in Fig. 2.

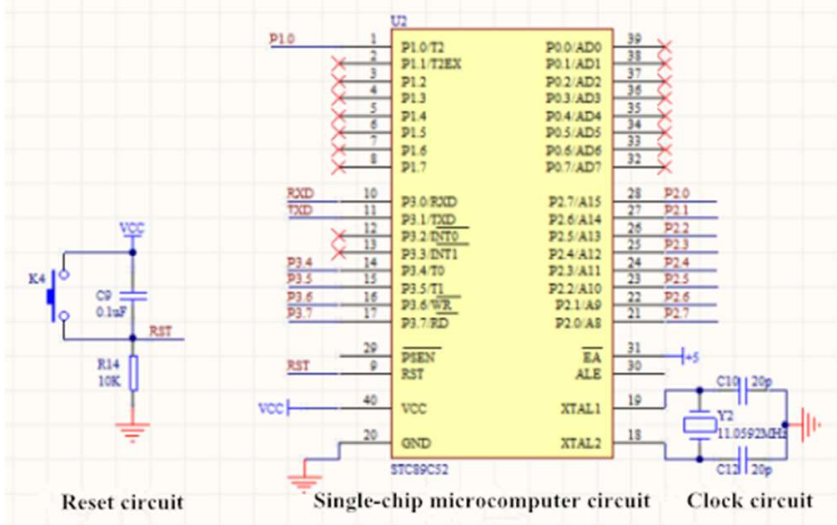

Figure 2. Minimal system circuit design of single chip microcomputer

\subsection{Design of photosensitive module circuit}

The system uses photoresistor to collect the light intensity signal, then transfers the collected analog signal to ADC0832 to digital signal, and then transmits it back to the single-chip microcomputer. The circuit diagram of the photoresistor in this system is shown in Fig. 4.

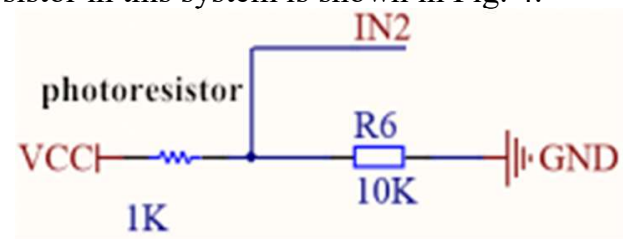

Figure 3. Circuit diagram of photoresistor

\subsection{Design of carbon dioxide detection module}

The carbon dioxide detection module adopts MG811 solid electrolyte sensor. The module uses the carbon dioxide gas sensing probe to collect information, which is processed by LM393 chip and transmitted to ADC0832 to convert digital information. The entire module has five pins: Power supply positive input Vcc, TTL signal output DOUT, analog signal output AOUT, power supply negative input GND, temperature compensation output TCM. The pins required for the design are Vcc, AOUT, and GND. The voltage of the analog signal output is $0-2 \mathrm{~V}$. The lower the concentration of carbon dioxide gas, the higher the voltage value of the output. The circuit design of the carbon dioxide detection module is shown in Fig. 3. Connect the AOUT pin to an input pin of the ADC0832.

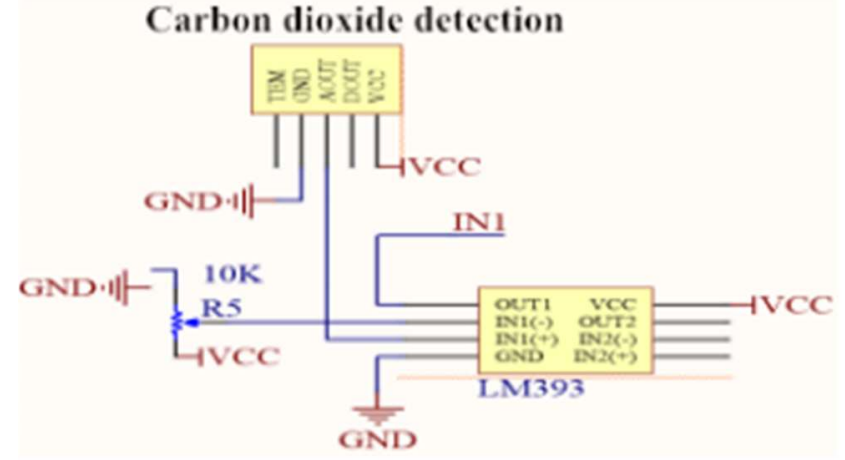

Figure 4. Circuit diagram of carbon dioxide detection module

\subsection{Design of A/D conversion circuit}

Since ADC0832 can be converted into two channels, the IN1 and IN2 pins of the chip are connected to the photoresistor and the OUT1 pin of the LM393, and the VCC and GND are connected to the power supply and the ground respectively. Since D0 and D1 are not used simultaneously with the single-chip microcomputer, it is possible to connect the two ports in parallel to the T1/P3.5 pin of the single-chip microcomputer and connect the CS pin WR/P3.6. Connect SCLK to the T0/p3.4 pin. The circuit diagram is shown in Fig. 5.

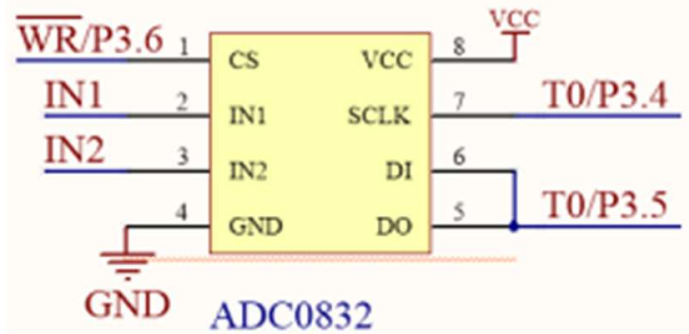

Figure 5. A/D conversion circuit

\subsection{Design of temperature and humidity detection module}

The temperature and humidity detection module uses the DHT11 temperature and humidity sensor, and the output signal is a digital signal. The OUT pin of the sensor can be directly connected to the single-chip microcomputer in the circuit. VCC and GND are connected to power and ground respectively. The circuit diagram is shown in Fig. 6.

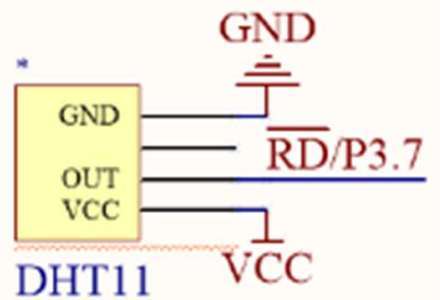

Figure 6. Circuit diagram of DHT11 temperature and humidity detection module 


\subsection{Design of $4^{*} 4$ matrix keyboard circuit}

The setting of the limited values in this system uses the matrix keyboard of $4 * 4$, and the circuit diagram is shown in Fig. 7. P1.0 P1.7 of single-chip microcomputer is connected with the matrix keyboard. A $1 \mathrm{k} \mathrm{A102J}$ resistor is connected in the middle to protect the circuit. This resistor is also referred to as a pull-up resistor in the circuit to ensure that the single-chip microcomputer input remains high when no buttons are pressed.
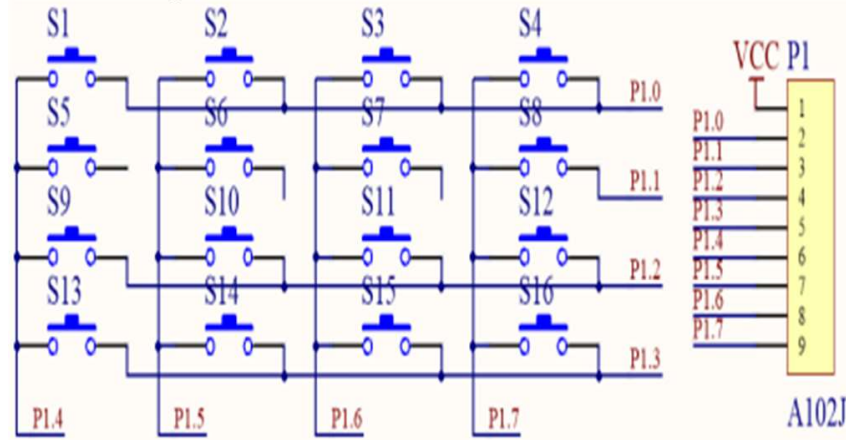

Figure 7. Circuit design of $4 * 4$ matrix keyboard

\subsection{Design of LCD display module circuit}

The display module uses an LCD1602 liquid crystal display. The circuit diagram of the display module is shown in Fig. 8. The D0-D7 of 1602 is connected to the P0.0-P0.7 pin of the single-chip computer, and an A102J pull-up resistor is added to ensure that the D0-D7 is effective at low level and plays a role in protecting the circuit. A variable resistor is connected to the V0 pin of the LCD screen to achieve the effect of independently adjusting the backlight brightness of the LCD screen. The P2.5-P2.7 of the single-chip computer connects three control bits of RS, RW and EN on the LCD screen.

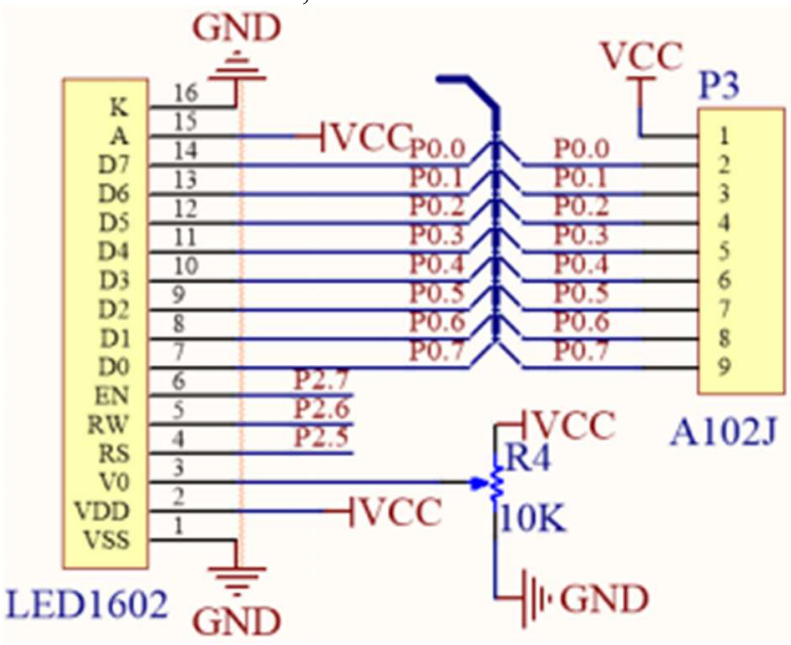

Figure 8. Design of LCD display module circuit

\subsection{Design of alarm module circuit}

The system uses an active buzzer, and the circuit diagram is shown in Fig. 9. The driving current of the active buzzer is about $25 \mathrm{~mA}$. An amplifier circuit is needed to drive the buzzer. The whole alarm circuit only needs a P2.0 port of the single-chip microcomputer.

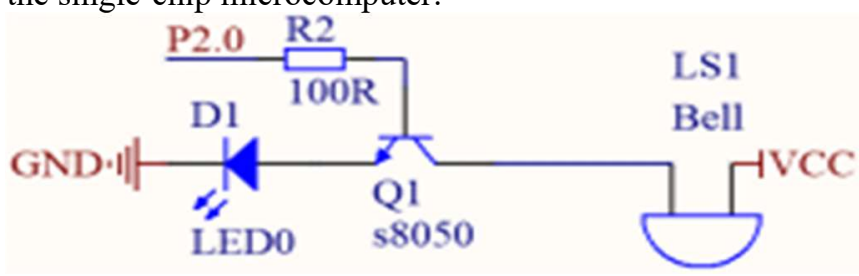

Figure 9. Design of alarm module circuit

\subsection{Design of execution module circuit}

The execution module in this system includes a fan and a light-emitting diode, which are respectively driven by $\mathrm{P} 2.2$ port and P2.1 port of the single-chip microcomputer. Since the current of the I/O port of the single-chip microcomputer cannot drive the fan to rotate, it is also necessary to have an amplifying circuit to increase the current to drive the fan to rotate. The LED can be directly connected to P2.2. The circuit diagram is shown in Fig. 10.

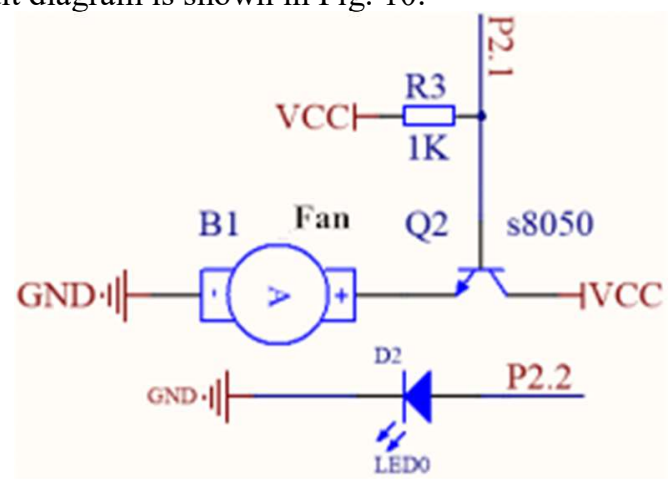

Figure 10. Design of fan and LED circuit

\section{System software design}

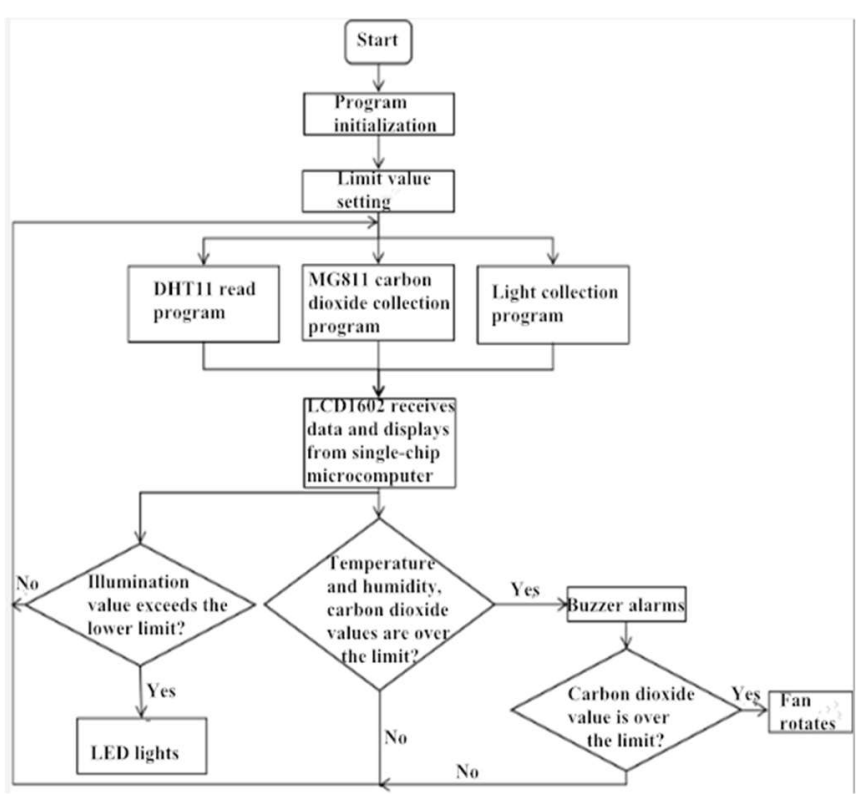

Figure 11. Overall flow chart of software design 
The block diagram of the system is shown in Fig. 11. After the system is powered on, the program will perform initialization work, and then the limit value can be set, after which the DHT11 read program starts executing. Since DHT11 has been subjected to the analog to digital process, it can directly read its digital quantity. The MG811 and the photoresistor output are analog quantities, so they must be input to the single-chip microcomputer after A/D conversion. The single-chip microcomputer compares all received digital quantities with the set value. When the light intensity reaches the set value, the LED will be automatically lit. When other parameters exceed the limit, the program will jump to the buzzer alarm and determine whether the carbon dioxide concentration is over-limit. If the concentration of carbon dioxide exceeds the limit, jump to the driver of the fan. If there is no value exceeding the limit, repeat the cycle program to display the collected data in real time.

\section{System debugging}

\subsection{Test environment}

Hot water bottles are used to raise the temperature around DHT11, and wet wipes are used to increase environmental humidity. The light test is covered with a thick paper bucket to test the function of the light complement. Burning a piece of paper in a sealed plastic bottle creates an environment with a high concentration of carbon dioxide. When the above-mentioned items are prepared, each function can be tested separately. Each function can be checked one by one to see if it works.

\subsection{Test results}

After testing each module, it is found that the system can achieve temperature and humidity detection, illumination and $\mathrm{CO}_{2}$ concentration detection. In the case of the over-limit value, the alarm and reminder can be issued at the first time, the fan can be switched on at the first time when the carbon dioxide concentration is over the limit, and the LED can change with the change of light intensity. The finished product is shown in Fig. 12.

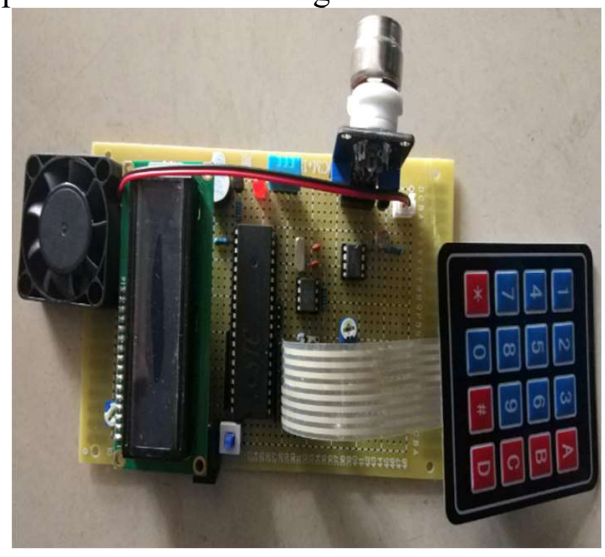

Fig. 12 Physical map

\section{Conclusion}

Intelligent monitoring system of chicken house designed in this paper uses a variety of sensors to collect environmental information of chicken house and display the collected information in real time. When the environmental parameters change beyond the set value, the fan and LED can be automatically controlled to improve the environment of chicken house. Experiments show that the system extracts several factors which can not be lacked in the growing environment of chickens for detection, making the system more universal and suitable for monitoring in many breeding environments.

\section{References}

1. Diao Zhigang, Yin Jia long, Zhu Xiaoning. Model Design of Aquaculture Environment Monitoring System Based on Multi-sensor Data Fusion[]]. Electronic Technology and Software Engineering, 2015 (01): 81-82.

2. Fu Qian. Design of Environmental Control System Based on Single Chip Microcomputer Poultry House[]]. Technology Innovation and Application. 2014(14).

3. Shen Chen. Design of Environment Monitoring System for Poultry Based on Wireless Sensor Network[D]. Anhui University of Science and Technology, 2017.

4. Feng Yuqian. Design and Development of Farm Information Collection System[D]. Shaanxi University of Science and Technology, 2016.

5. Xu Chenxi, Li Lihua, Huang Mengxuan, etc. Design and Application of Environmental Monitoring and Alarm System for Chicken House Parametres Based on the Internet[]]. Acta Ecologae Animalis Domastici, 2017, 38(10): 43-50.

6. He Wenzhao. Design of Chicken House Environment Monitoring System Based on Single Chip Microcomputer[D]. Inner Mongolia Agricultural University, 2015. 